\title{
Pesquisa
}

\section{Acesso aos serviços de saúde bucal por pacientes com Paralisia Cerebral no município de Campina Grande - PB}

Access to oral health services by patients with Cerebral Palsy in the city of Campina Grande - PB

Rênnis Oliveira da Silva1; Lays Nóbrega Gomes²; Clara Regina Duarte Silva ${ }^{3}$; Andreia Medeiros Rodrigues Cardoso4; Alessandro Leite Cavalcanti5; Wilton Wilney Nascimento Padilha ${ }^{6}$.

${ }^{1}$ Graduando do Curso de Odontologia, Universidade Federal da Paraíba, João Pessoa-PB ${ }^{2}$ Mestranda em Reabilitação Oral, Faculdade de Odontologia de Araraquara, Araraquara-SP ${ }^{3}$ Cirurgiã-Dentista, Universidade Federal da Paraíba, João Pessoa-PB

${ }^{4}$ Professora Dra ${ }^{\text {. }}$ do Curso de Odontologia, Centro Universitário de João Pessoa, João Pessoa-PB ${ }^{5}$ Professor Dr. do Curso de Odontologia, Universidade Estadual da Paraíba, Campina Grande-PB ${ }^{6}$ Professor Dr. do Curso de Odontologia, Universidade Federal da Paraíba, João Pessoa-PB

Endereço para correspondência:

Wilton Wilney Nascimento Padilha, Programa de Pós-graduação em Odontologia, Centro de Ciências da Saúde, Universidade Federal da Paraíba - Campus I. Wilton.padilha@yahoo.com.br

\section{Resumo}

Introdução: No Brasil, as unidades básicas de saúde devem ofertar e garantir atendimento a pacientes com necessidades especiais. Objetivo: Verificar a forma de acesso aos serviços de saúde bucal por pacientes com Paralisia Cerebral (PC) e seus fatores associados. Metodologia: Estudo transversal realizado na APAE do município de Campina Grande-PB, com 80 crianças e adolescentes com diagnóstico de PC. Os cuidadores primários responderam um formulário de 50 perguntas relacionadas a características socioeconômicas, sistêmicas e de acesso aos serviços de saúde bucal. Os dados foram analisados de forma descritiva e com análise de Poisson $(\alpha<0,05)$. Resultados: $81,3 \%$ da amostra já foi ao dentista, sendo $62,4 \%$ no serviço público. A primeira consulta odontológica foi realizada por motivo preventivo para $31,3 \%$ dos pacientes. $60 \%$ relataram ter dificuldades de ir ao dentista, $47,5 \%$ informaram que essa dificuldade ocorre pela baixa oferta de profissionais capacitados. $\mathrm{Na}$ análise de Poisson o tempo de última consulta em até dois anos foi associado a: renda familiar menor que $\mathrm{R} \$ 900,00$ (RP 1,32; IC95\%=1,11-1,57); boa percepção de saúde geral (RP 2,09; IC95\%=1,43-3,05); ausência de dor de dente nos últimos seis meses (RP 0,67; IC95\%=0,56-0,81); baixa oferta de dentista (RP 1,48; IC95\%=1,02-2,14). Conclusão: As crianças e adolescentes com paralisia cerebral já foram ao dentista, embora encontrem dificuldades para o acesso devido à baixa oferta de profissionais. $O$ tempo de última consulta em até dois anos foi associado à renda familiar, percepção de saúde geral, ausência de dor de dente.

Descritores: Paralisia Cerebral. Acesso aos Serviços de Saúde. Cuidado da Criança. 


\begin{abstract}
Introduction: In Brazil, how basic health units should be treated and ensure care for special patients. Objective: To verify the form of access to oral health services by patients with Cerebral Palsy (PC) and their associated factors. Methodology: A cross-sectional study conducted at APAE in the city of Campina Grande, Brazil, with 80 children and adolescents diagnosed with PC. Caregivers receive a form of 50 sessions related to socioeconomic, systemic and access to oral health services. The data were analyzed descriptively and with the Poisson analysis $(\alpha<0.05)$. Results: $81.3 \%$ of the sample went to the dentist, $62.4 \%$ in the public service. The first dental appointment was performed due to preventive reasons for $31.3 \%$ of the patients. $60 \%$ reported the dentist's difficulties, $47.5 \%$ reported that this difficulty was reduced by the availability of trained professionals. The Poisson analysis had a minimum value of $\mathrm{R} \$ 900.00(\mathrm{RP} 1.32,95 \% \mathrm{Cl}=1.11-1.57$ ); good general health perception (PR 2.09, 95\% Cl=1.43-3.05); no pain at six months (RP $0.67,95 \% \mathrm{Cl}=0.56-0.81)$; lower dentist supply $(\mathrm{RP} 1.48,95 \% \mathrm{Cl}=1.02-2.14)$. Conclusion: Children and adolescents with cerebral palsy have already gone to the dentist, although they find it difficult to access due to an offer of work. Consultation time in two years was associated with family income, general health perception, and absence of toothache.
\end{abstract}

Keywords: Cerebral Palsy. Health Services Accessibility. Child Care.

\title{
INTRODUÇÃO
}

A Paralisia Cerebral (PC) é um grupo de distúrbios que afetam o desenvolvimento do movimento e da postura. A PC causa limitações de atividades que são atribuídas a distorções não progressivas e que ocorreram no desenvolvimento fetal ou do cérebro infantil1.

Em uma revisão sistemática foi observado que crianças com Paralisia Cerebral possuem maior frequência de cárie, alterações periodontais, traumatismos dentários e uma higiene bucal mais deficiente que as sem essa condição ${ }^{2}$, sendo esses problemas associados a características socioeconômicas ${ }^{3}$.

No Brasil, as unidades básicas de saúde estão aptas e devem se organizar e definir ações para ofertar e garantir atendimento prioritário a esses pacientes, no âmbito da atenção primária ${ }^{4}$.

Em casos de maior complexidade, pacientes com PC devem ser encaminhadas para os Centros de Especialidades Odontológicas que foram implantados por meio da portaria № 599 de 23 de março de 2006. Essa portaria define que esses estabelecimentos de saúde possuam atendimento a portadores de necessidades especiais ${ }^{5}$. 
Porém, em estudos realizados com todas as faixas etárias, o baixo status socioeconômico e o grau de escolaridade baixo, são observados como uma barreira para o acesso aos serviços de saúde ${ }^{6,7}$.

Sendo assim, o objetivo desse estudo foi verificar a forma de acesso aos serviços de saúde bucal por crianças e adolescentes com paralisia cerebral (CAPC), e seus fatores associados, no município de Campina Grande - PB.

\section{METODOLOGIA}

Pesquisa transversal de abordagem indutiva, com procedimento analítico-descritivo e técnica de observação direta intensiva ${ }^{8}$.

Esta pesquisa foi realizada na Associação de Pais e Amigos dos Excepcionais (APAE) da cidade de Campina Grande - Paraíba, Brasil, no ano de 2015. A cidade possui aproximadamente 410 mil habitantes e um Índice de Desenvolvimento Humano de 0,72.

A APAE é uma instituição filantrópica que atua nas áreas de educação, saúde e assistência social. Na área de saúde, atua para promover a atenção integral à pessoa com deficiência, em todo o seu ciclo de vida.

A instituição conta com 97 pacientes regularmente matriculados com diagnóstico médico clínico de paralisia cerebral (CID10 - G80) entre 2 e 18 anos de idade. A amostra foi composta por 80 desses pacientes (taxa de resposta 82,5\%).

A coleta dos dados foi realizada através da aplicação, face a face com os cuidadores primários. Foi utilizado um formulário de 50 perguntas que investiga as características socioeconômicas, comportamentais (hábitos alimentares, de higiene bucal e deletérios) e de acesso ao serviço de saúde bucal dos pacientes com paralisia cerebral.

Foi definido como cuidador primário aquele que apresentava 18 anos ou mais e era responsável pelas tomadas de decisões e pela realização das atividades diárias das CAPC.

Os dados foram tabulados com o Software Statistical Package for the Social Sciences (SPSS para Windows, versão 20.0, SPSS Inc, Chicago, IL, EUA) e analisados de forma descritiva e por meio da Regressão de Poisson $(\alpha<0,05)$.

Para a Regressão de Poisson as CAPC que nunca foram ao dentista, foram classificadas em tempo de consulta maior que dois anos.

Esta pesquisa foi aprovada pelo Comitê de Ética em Pesquisa da Universidade Estadual da Paraíba (CAAE 20215413.4.0000.5187) e seguiu as diretrizes éticas recomendadas pela legislação 
brasileira e internacional, para isso todos os participantes assinaram o Termo de Consentimento Livre e Esclarecido.

\section{RESULTADOS}

As CAPC eram do sexo masculino $(52,5 \%, n=42)$ e feminino $(47,5 \%, n=38)$, com idade entre 2 e 11 anos $(61,2 \%, n=49)$ e 12 a 18 anos $(38,7 \%, n=31)$, com tetraparesia $(56,3 \%, n=45)$ e espásticos $(75,0 \%, \mathrm{n}=60)$.

A Tabela 1 descreve a utilização dos serviços odontológicos por CAPC, sob a perspectiva do cuidador em valores absolutos e percentuais.

\begin{tabular}{|c|c|c|}
\hline \multirow{3}{*}{ Variáveis } & \multirow{2}{*}{\multicolumn{2}{|c|}{ Frequência }} \\
\hline & & $\%$ \\
\hline & \multicolumn{2}{|c|}{ Foi ao dentista } \\
\hline $\operatorname{Sim}$ & 65 & 81,3 \\
\hline Não & 15 & 18,8 \\
\hline \multicolumn{3}{|c|}{ Motivo da primeira consulta odontológica } \\
\hline Revisão ou prevenção & 25 & 31,3 \\
\hline Dor & 9 & 11,3 \\
\hline Cárie & 8 & 10,0 \\
\hline Exodontia de dentes decíduos & 5 & 6,3 \\
\hline Outros motivos & 18 & 22,3 \\
\hline Não se aplica & 15 & 18,8 \\
\hline \multicolumn{3}{|c|}{ Motivo da última consulta odontológica } \\
\hline Dentes estragados & 20 & 25,0 \\
\hline Consulta preventiva & 18 & 22,5 \\
\hline Dor de dente & 12 & 15,0 \\
\hline Outros & 14 & 17,5 \\
\hline Não se aplica & 11 & 23,3 \\
\hline \multicolumn{3}{|c|}{ Recursos adicionais utilizados no atendimento ambulatorial } \\
\hline Participação ativa do cuidador & 40 & 50,0 \\
\hline Nenhum & 13 & 16,3 \\
\hline Outros & 11 & 13,9 \\
\hline Não se aplica & 16 & 19,8 \\
\hline \multicolumn{3}{|c|}{ Tempo decorrido desde a última consulta odontológica } \\
\hline Até 2 anos & 51 & 63,8 \\
\hline Mais de 2 anos & 29 & 36,6 \\
\hline \multicolumn{3}{|l|}{ Setor de atendimento } \\
\hline Público & 50 & 62,4 \\
\hline Privado & 15 & 18,8 \\
\hline Não se aplica & 15 & 18,8 \\
\hline \multicolumn{3}{|c|}{ Local de atendimento da primeira consulta odontológica } \\
\hline PSF & 20 & 25,0 \\
\hline Hospital & 13 & 16,3 \\
\hline Consultório Privado & 15 & 18,8 \\
\hline Outros & 17 & 21,1 \\
\hline
\end{tabular}




\begin{tabular}{|c|c|c|}
\hline Não se aplica & 15 & 18,8 \\
\hline \multicolumn{3}{|c|}{ Transporte utilizado para deslocamento } \\
\hline Carro próprio & 17 & 21,3 \\
\hline Transporte da prefeitura & 17 & 21,3 \\
\hline Outros & 32 & 39,9 \\
\hline Não se aplica & 14 & 17,5 \\
\hline \multicolumn{3}{|c|}{ Apresenta dificuldade de ir ao dentista } \\
\hline $\operatorname{Sim}$ & 48 & 60,0 \\
\hline Não & 32 & 40,0 \\
\hline \multicolumn{3}{|c|}{ Tipo de dificuldade para utilizar o serviço odontológico } \\
\hline Baixa oferta de dentista & 38 & 47,5 \\
\hline Demora no agendamento & 8 & 10,0 \\
\hline Indisponibilidade de anestesia geral & 2 & 2,5 \\
\hline Não se aplica & 32 & 40,0 \\
\hline Total & 80 & 100 \\
\hline
\end{tabular}

A Tabela 02 apresenta a distribuição das CAPC nos modelos bi e multivariado de regressão de Poisson para utilização de serviços odontológicos.

Tabela 2. Distribuição das crianças e adolescentes com PC nos modelos bi e multivariado de regressão de Poisson para utilização dos serviços odontológicos e as variáveis independentes.

\begin{tabular}{|c|c|c|c|c|c|c|}
\hline \multirow{3}{*}{ Variável } & \multicolumn{2}{|c|}{$\begin{array}{c}\text { Tempo da última } \\
\text { consulta }\end{array}$} & \multicolumn{2}{|c|}{ Bivariada } & \multicolumn{2}{|c|}{ Multivariada } \\
\hline & Até 2 anos & $\begin{array}{l}\text { Nunca foi } \\
\text { ou foi há } \\
\text { mais de } 2\end{array}$ & \multicolumn{2}{|c|}{ Não ajustado RP* } & \multicolumn{2}{|c|}{ Ajustado RP* } \\
\hline & $\mathrm{n}(\%)$ & $\mathrm{n}(\%)$ & $\mathrm{p}$-valor & $(95 \% \mathrm{IC})$ & $\begin{array}{c}p- \\
\text { valor }\end{array}$ & $(95 \%$ IC) \\
\hline \multicolumn{7}{|l|}{ Sexo } \\
\hline Masculino & $28(66.7)$ & 14(33.3) & 0.568 & $0.956(0.81-1.11)$ & - & - \\
\hline Feminino & $23(60.5)$ & 15(39.5) & & 1.0 & - & - \\
\hline \multicolumn{7}{|l|}{ Idade (anos) } \\
\hline $2 \mathrm{a} 11$ & $32(65.3)$ & $17(34.7)$ & 0.716 & $0.971(0.82-1,13)$ & - & - \\
\hline 12 a 18 & 19(61.3) & $12(38.7)$ & & 1.0 & - & - \\
\hline \multicolumn{7}{|l|}{ Renda familiar } \\
\hline$\leq 900,00$ & $22(52.4)$ & $20(47.6)$ & 0.021 & $1.194(1.02-1.37)$ & 0.002 & $\begin{array}{c}1.322(1.11- \\
1.57)\end{array}$ \\
\hline$>900,00$ & 29(76.3) & $9(23.7)$ & & 1.0 & & 1.0 \\
\hline \multicolumn{7}{|c|}{ Escolaridade do cuidador (em anos) } \\
\hline$\leq 8$ & $21(60.0)$ & $14(40.0)$ & 0.538 & $1.050(0.89-1.22)$ & - & - \\
\hline$>8$ & $30(66.7)$ & 15(33.3) & & 1.0 & - & - \\
\hline \multicolumn{7}{|c|}{ Percepção da saúde geral da CAPC } \\
\hline Boa & $48(62.3)$ & $29(37.7)$ & 0.000 & $1.377(1.27-1.48)$ & 0.000 & $\begin{array}{c}2.090(1.43- \\
3.05)\end{array}$ \\
\hline Ruim & $3(100.0)$ & $0(0)$ & & 1.0 & 0 & 1.0 \\
\hline \multicolumn{7}{|c|}{ Percepção da saúde bucal da CAPC } \\
\hline De ótima a boa & $38(64.4)$ & $21(35.6)$ & 0.838 & $0.982(0.82-1.17)$ & - & - \\
\hline De ruim a péssima & 13(61.9) & 8(38.1) & & 1.0 & - & - \\
\hline \multicolumn{7}{|c|}{ Presença de dor de dente nos últimos 6 meses } \\
\hline Não & $49(66.2)$ & $25(33.8)$ & 0.073 & $0.803(0.63-1.02)$ & 0.000 & $0.675(0.56-0.81)$ \\
\hline Sim & $2(33.3)$ & $4(66.7)$ & & & & 1.0 \\
\hline
\end{tabular}


Habilidade de comunicação Normal

$\begin{array}{cccccc}5(83.3) & 1(16.7) & 0.027 & 1.429(1.04-1.95) & 0.000 & \begin{array}{c}2.186(1.58- \\ 3.01)\end{array} \\ 12(60.0) & 8(40.0) & 0.407 & 1.124(0.85-1.48) & 0.016 & \begin{array}{c}1.353(1.05- \\ 1.73)\end{array} \\ 31(68.9) & 14(31.1) & 0.231 & 1.200(0.89-1.61) & 0.043 & \begin{array}{c}1.369(1.01- \\ 1.85)\end{array} \\ 3(33.3) & 6(66.7) & & 1.0 & & 1.0 \\ 32(66.7) & 16(33.3) & 0.506 & 0.948(0.81-1.10) & - & - \\ 19(59.4) & 13(40.6) & & 1.0 & - & - \\ 25(65.8) & 13(34.2) & 0.000 & 1.778(1.45-2.17) & 0.038 & 1.482(1.02- \\ 7(87.5) & 1(12.5) & 0.137 & 1.193(0.94-1.50) & 0.502 & 1.080(0.84- \\ & & & & & 1.39) \\ 0 & 2(100) & & 1.0 & & 1.0\end{array}$

$\mathrm{Na}$ análise bivariada, a variável utilização dos serviços odontológicos nos últimos dois anos foi associada à: renda, percepção de saúde geral das CAPC, dor de dente nos últimos seis meses, habilidade de comunicação e principal dificuldade enfrentada na busca do serviço $(p<0.2)$. Essas variáveis foram incorporadas no modelo multivariado.

No modelo multivariado da regressão de Poisson para última consulta odontológica por CAPC nos últimos dois anos, foram encontradas associações com: a renda familiar me-nor que $R \$ 900,00(R P=1,32 ; I C 95 \%=1,11-1,57)$, a percepção de saúde geral boa ( $R P=2,09$; IC95\%=1,43$3,06)$, a ausência de dor de dente $(\mathrm{RP}=0,67 ; \mathrm{IC} 95 \%=0,56-0,81)$, a habilidade de comunicação normal $(R P=2,18$; IC95\%=1,58-3,01) e deficiência leve $(R P=1,35 ; I C 95 \%=1,05-1,73)$, e a principal dificuldade a baixa oferta de dentistas habilitados $(\mathrm{RP}=1,48 ; 1,02-2,14)$.

\section{DISCUSSÃO}

Nesse estudo observou-se que a amostra é equivalente em relação ao sexo, assim como em estudo realizado na APAE do município de João Pessoa-PB (MEDEIROS et al., 2017). O perfil espástico $(75,0 \%)$ foi semelhante ao encontrado $(70,7 \%)$ em estudo realizado em Recife-PE 9 .

Entre as CAPC observou-se que $81,3 \%$ já passaram por atendimento odontológico, resultado semelhante foi encontrado em estudo realizado no município de João Pessoa-PB com crianças com deficiência motora de 2 a 12 anos, onde 69,0\% havia passado por atendimento ${ }^{10}$.

Para primeira consulta odontológica o motivo predominante foi revisão/prevenção, assim como descrito em estudo anterior ${ }^{10}$. Para última consulta, a prevenção também tem destaque 
dividindo espaço com "dentes estragados". Esse perfil preventivo pode ser explicado pela amostra ser atendida em uma instituição que visa o cuidado integral dos pacientes.

A participação ativa do cuidador foi relatada pela maioria dos entrevistados como um recurso adicional utilizado no atendimento odontológico, isso pode ser explicado pelos espasmos musculares que acometem os pacientes com PC.

O tempo decorrido desde a última consulta odontológica foi de até 2 anos, esse resultado também foi visto em João Pessoa - $\mathrm{PB}^{10}$.

A primeira consulta odontológica foi realizada em PSF, isso mostra cumprimento do que foi preconizado pelo Ministério da Saúde para a atenção básica que garante atendimento a pacientes com necessidades especiais.

Foi relatado por $60,0 \%$ da amostra a dificuldade de ir ao dentista, assim como em estudo de Cardoso $^{10}$. O contrário é encontrado em estudo com escolares em um município no interior da Paraíba onde $79,3 \%$ dos pais relataram não encontrar dificuldades para acesso aos serviços odontológicos ${ }^{7}$.

Esse contraste pode ser explicado pela dificuldade dos cuidadores das CAPC's encontrarem atendimento devido à baixa oferta de dentista qualificados para o atendimento, dificuldade essa também encontrada em outro estudo? .

A associação encontrada entre a renda familiar menor que $\mathrm{R} \$ 900,00$ com nunca ter ido ou ter ido há mais de dois anos no dentista, pode ser explicada pela baixa oferta de dentistas capacitados no serviço público e a dificuldade de custear o serviço privado.

A percepção de saúde geral boa foi associada a ter ido ao dentista nos últimos dois anos, essa associação pode estar relacionada por esse ser um grupo de pacientes que sejam mais cuidados em relação a sua saúde como um todo.

A ausência de dor de dente nos últimos seis meses, foi associada com ir ao dentista nos últimos dois anos, essa associação pode ser explicada por esse ser um grupo que visita o dentista com maior frequência e acaba por não desenvolver problemas maiores.

A associação encontrada entre os pacientes que visitaram o dentista nos últimos dois anos e a dificuldade de utilização dos serviços odontológicos devido à baixa oferta de dentista, mostra problemas no acesso que podem ser ocasionados por falta de profissionais preparados para 0 atendimento, unidade de saúde distante do endereço de residência. 


\section{CONCLUSÃO}

As crianças e adolescentes com paralisia cerebral já foram ao dentista, embora encontrem dificuldades para o acesso devido à baixa oferta de profissionais. O tempo de última consulta em até dois anos foi associado à renda familiar, percepção de saúde geral, ausência de dor de dente, habilidade de comunicação e baixa oferta de dentista.

\section{REFERÊNCIAS}

1. Rosenbaum P, Paneth N, Leviton A, Goldstein M, Bax M, Damiano D, et al. A report: The definition and classification of cerebral palsy April 2006. Dev Med Child Neurol. 2007;49(109):8-14.

2. Diéguez-Pérez M, de Nova-García MJ, Mourelle-Martínez MR, Bartolomé-Villar B. Oral health in children with physical (Cerebral Palsy) and intellectual (Down Syndrome) disabilities: Systematic review I. J Clin Exp Dent. 2016;8(3):337-343.

3. Cardoso A, Gomes L, Silva C, Soares R, de Abreu M, Padilha W, et al. Dental Caries and Periodontal Disease in Brazilian Children and Adolescents with Cerebral Palsy. Int J Environ Res Public Health. 2014;12(1):335-353.

4. Brasil MDS. Cadernos de Atenção Básica - Saúde Bucal. Secretaria de Atenção à Saúde. Departamento de Atenção Básica. Saúde Bucal / Ministério da Saúde, Secretaria de Atenção à Saúde, Departamento de Atenção Básica. 2006. 92 p. il. - (Cadernos de Atenção Básica, 17).

5. Ministério da Saúde. PORTARIA № 599 DE 23 DE MARÇO DE 2006. 2006.

6. Baldani $\mathrm{MH}$, Brito $\mathrm{WH}$, Lawder JA de C, Mendes YBE, Silva F de FM da, Antunes JLF. Determinantes individuais da utilização de serviços odontológicos por adultos e idosos de baixa renda. Rev Bras Epidemiol. 2010;13(1):150-162.

7. Paredes SO, Fernandes JRL, Fernandes JMF de A, Menezes VA. Utilização dos serviços odontólogicos por pré-escolares em um município de pequeno porte do Estado da Paraíba. Rev Odontol da UNESP. 2015;44(3):181-187.

8. Marconi M, Lakatos E. Fundamentos de metodologia científica. Editora Atlas S. A. 2003. 310 p.

9. Lemos ACO, Katz CRT. Condições de saúde bucal e acesso ao tratamento odontológico de pacientes com paralisia cerebral atendidos em um centro de referência do Nordeste - Brasil. Rev CEFAC. 2012;14(5):861-871.

10. Cardoso AMR, Brito DBA, Alves VF, Padilha WWN. O acesso ao cuidado em saúde bucal para crianças com deficiência motora: Perspectivas dos cuidadores. Pesqui Bras Odontopediatria Clin Integr. 2011;11(4):593-599. 